\title{
Skin color variation: A study on Eastern and North East India
}

\author{
Sumit Maitra1, Diptendu Chatterjee ${ }^{2}$, Arup Ratan Bandyopadhyay ${ }^{3}$ \\ ${ }^{1}$ Research Student, Department of Anthropology, University College of Science, University of Calcutta, 35, Ballygunge \\ Circular Road, Kolkata - 700019, India, ${ }^{2}$ Assistant Professor (Grade III), Department of Anthropology, University \\ College of Science, University of Calcutta, 35, Ballygunge Circular Road, Kolkata - 700019, India, ${ }^{3}$ Professor, \\ Department of Anthropology, University College of Science, University of Calcutta, 35, Ballygunge Circular Road, \\ Kolkata - 700019, India
}

A B S TR A C T

Background: Skin pigmentation is one of the most variable phenotypic traits and most noticeable of human polymorphisms. Skin pigmentation in humans is largely determined by the quantity and distribution of the pigment melanin. The literature review on skin color variation revealed a few works on skin pigmentation variation has been conducted in India from Southern, Western and Northern part. Aims and Objectives: To best of the knowledge, the present discourse is the first attempt to understand skin color variation from Eastern and North Eastern part of India among three populations. Materials and Methods: The present study consisted of 312 participants from Chakma and Tripuri groups of Tripura, North East India, and participants from Bengalee Hindu caste population from West Bengal. Skin color was measured by Konica Minolta CR-10 spectrophotometer which measures and quantifies the colors with a 3D color space (CIELAB) color space created by 3 axes. All the skin color measurements from each participant were taken from unexposed (underarm) left and right to get a mean and exposed (forehead) to sunlight. Results: The distribution of skin color variation among the three populations demonstrated significant $(p<0.05)$ difference in lightness for unexposed and exposed indicating lightness in unexposed area. Furthermore, the present study revealed significant difference $(p<0.05)$ in skin color among the ethnic groups across the body location and all three attributes (lightness, redness and yellowness). Conclusion: Generally, skin color variation may be elucidated by two main factors: individual differences in lightness and yellowness and by and large due to ethnicity, where diversity in redness is due to primarily due to different body locations. Variation in lightness have more characteristic probability reflecting the large influence of environmental factors such as exposure to sun. The present study first time reports the wide range of quantitative skin color variation among the three ethnic groups from Eastern and North East India and highest yellowness $\left(b^{*}\right)$ among the population from North East India.

Key words: Skin colour; Exposed; Unexposed; Eastern India; North East India

\section{SKIN COLOR VARIATION: A STUDY ON EASTERN AND NORTH EAST INDIA}

The origin of humans and the migration to inhabit different corners of the world has been a subject matter of various research and considerable debate for biologists, archaeologists and anthropologists. Researches on evolutionary biology amidst one of the key themes are to understand human adaptations and the various adaptive traits those have acquired during these years. There are a few distinct physical traits that differentiate us from apes, ${ }^{1,2}$ but at the same time humans themselves encompass huge within species phenotypic diversity. ${ }^{3}$ The diverse array of phenotypes that are currently visible across the world seems to be results of adaptations due to diverse selective pressures and/or evolutionary forces acting at different time and space in the evolutionary history of human. 
The discourse of genetic architecture of adaptive traits, yielded results towards common umbrella of complex traits, as they are governed by both genes as well as environment and therefore, unraveling them is a crucial step. On the other hand, the environmental influences are not always constant in time and space has often changed their environments by migration. Furthermore, culture and technology also intensely influence the evolution of phenotypic traits. ${ }^{4}$

Variation in skin color is the most conspicuous of human polymorphisms. Since the mid- $18^{\text {th }}$ century, skin color has been the one of most important morphological traits used to define human groups, including variously named varieties, races, and ethnic groups. The latter part of the $20^{\text {th }}$ century revealed that human skin reflectance (as a measure of skin pigmentation) was highly associated with latitude as one of the surrogates for Ultra Violet Radiation (UVR) than with temperature, humidity, or altitude. ${ }^{5,6}$ Human skin pigmentation is the product of two clines produced by natural selection to adjust levels of constitutive pigmentation to levels of UVR. One cline was generated by high UVR near the equator and led to the evolution of dark, photoprotective, eumelanin-rich pigmentation. The other was produced by the requirement for UVB photons to sustain cutaneous photosynthesis of vitamin D3 in low-UVB environments, and resulted in the evolution of depigmented skin. As hominids dispersed outside of the tropics, they experienced different intensities and seasonal mixtures of UVA and UVB. ${ }^{7}$

India, occupying the centre-stage of Palaeolithic and Neolithic migrations, and somewhat has been underrepresented in genome-wide studies of variation. ${ }^{8}$ Being at the cross-roads of migration, Indian populations have undergone complex and ancient admixture events over a long period ${ }^{9}$ and have been the melting-pot of disparate ancestries originating from different parts of Eurasia and South-East Asia. ${ }^{10}$ Although the date of entry of modern humans into India remains uncertain but it is reasonable to consider by the middle Paleolithic period $(50,000-20,000$ years before present, humans appear to have spread onto many parts of India. Contemporary ethnic India is a land of enormous genetic, cultural, and linguistic diversity. A more recent study ${ }^{10}$ exploring Indian genomic diversity demonstrated four major ancestral genetic components in mainland India that included four dominant ancestries in populations from mainland India: Ancestral North-Indian (ANI), Ancestral South-Indian (ASI), Ancestral Tibeto-Burman (ATB) and Ancestral Austro-Asiatic (AAA).

The literature review on skin color variation revealed a few works on skin pigmentation variation has been conducted in India from Southern part ${ }^{11}$ and Western and Northern part. ${ }^{12,13}$ To best of the knowledge, the present discourse is the first attempt to understand skin color variation from Eastern and North Eastern part of India among three populations.

\section{MATERIALS AND METHODS}

To achieve the purpose male participants from two Tibeto-Burman speaker endogamous ethnic populations (Chakmas - the migrant group and Tripuris - the aboriginal group) of North East India (Tripura) and Indo Aryan Speaker Bengalee population of West Bengal, India have been undertaken. The present study consisted of 105 participants from Chakma group and 103 from Tripuri group of Tripura along with 104 participants from Bengalee Hindu caste population from West Bengal. Verbal and/or Written consent was obtained from each participant prior to study. Skin color measurements were taken with the help of Konica Minolta CR-10 spectrophotometer following the instruction manual. It is a true colorimeter which provides the quantitative measures of a color and also the differences between colors. The instrument measures and quantify the colors with a 3D color space which is known as CIELAB color space, developed by CIE. ${ }^{14,15}$ In this 3 Dimensional space $L^{*}$ axis denotes the darkness or Lightness of a particular color. $\mathrm{a}^{*}$ axis denotes the Red-Green color space and $\mathrm{b}^{*}$ denotes the Yellow-Blue color space. A color is measured with the help of the co-ordinates created by these 3 axes. All the skin color measurements from each participant were taken from unexposed (underarm) left and right to get a mean and exposed (forehead) to sunlight. Obtained data were analyzed for descriptive and inferential statistics in appropriate places by SPSS (version 20.0). Cut off was set as $\mathrm{p}=0.05$.

\section{RESULTS AND DISCUSSION}

To evaluate skin color variation for each of the ethnic groups, the grand mean for each ethnic group was calculated by averaging each of the three attributes $\left(\mathrm{L}_{\mathrm{N}}^{*} ; \mathrm{a}_{\mathrm{M}}^{*} ; \mathrm{b}_{\mathrm{N}}^{*}\right)$ across the entire sample; then for each of the individuals the deviation from the group mean is calculated for all three attributes. The deviation of exposed and unexposed skin color has been calculated as $\delta \mathrm{E}$. The distribution of skin color variation among the three populations presented in the Table 1, demonstrated significant $(\mathrm{p}<0.05)$ difference in lightness for unexposed and exposed area among all the studied population indicating significant $(\mathrm{p}<0.05)$ lightness in unexposed area. The mean lightness (unexposed and unexposed) value was highest in the Bengalee. Similarly, for both the exposed and unexposed area redness $\left(\mathrm{a}^{*}\right)$ Bengalee demonstrated highest 


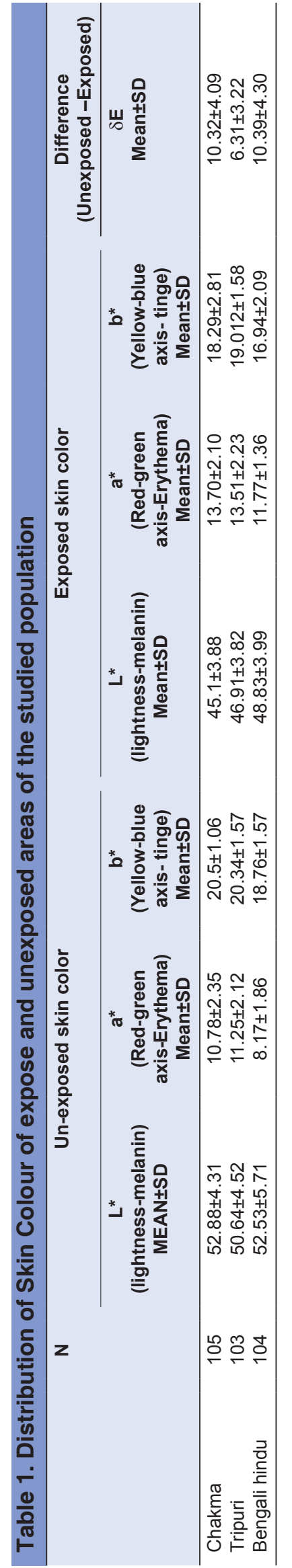

\begin{tabular}{|c|c|c|}
\hline Category & $\mathbf{F}$ & $p$ \\
\hline \multicolumn{3}{|l|}{ Unexposed skin color } \\
\hline L* (lightness-melanin) & 3.655 & 0.027 \\
\hline$a^{*}($ Red-green axis-Erythema) & 43.150 & 0.000 \\
\hline $\mathrm{b}^{*}($ Yellow-blue axis- tinge $)$ & 30.085 & 0.000 \\
\hline \multicolumn{3}{|l|}{ Exposed skin color } \\
\hline L* (lightness-melanin) & 14.555 & 0.000 \\
\hline$a^{*}$ (Red-green axis-Erythema) & 21.233 & 0.000 \\
\hline b* (Yellow-blue axis- tinge) & 15.989 & 0.000 \\
\hline
\end{tabular}

value. So far the yellowness dimension ( $b^{*}$ ) Chakmas and Tripuris demonstrated significant $(\mathrm{p}<0.05)$ wide variation from Bengalee for both the exposed and unexposed areas. Examination on both the exposed and unexposed skin color pigmentation (Table 1)between the populations with regard to the lightness $\left(\mathrm{L}^{*}\right)$ Red-green axis $\left(\mathrm{a}^{*}\right)$ and YellowBlue $\left(b^{*}\right)$ revealed significant $(p<0.05)$ difference among the Chakmas, Tripuris and Bengalee Hindu caste groups as well. Furthermore the deviation $(\delta \mathrm{E})$ of exposed and unexposed part revealed least deviation among the Tripuri $(\delta \mathrm{E}=6.31)$, than the Chakma $(\delta \mathrm{E}=10.32)$ and Bengalee $(\delta \mathrm{E}=10.39)$.

To evaluate how ethnicity and body location affects skin color, two ways ANOVA was performed (Table 2) to understand ethnic group variation and body location (exposed and unexposed). The present study revealed significant $(p<0.05)$ skin color variation among the ethnic groups in all three appearance attributes (lightness, redness and yellowness). Examination on the tanning effect in exposed area objectively measured by Red-green axisErythema ${ }^{16}$ demonstrated significantly $(\mathrm{p}<0.05)$ higher than unexposed area for all the group of population.

\section{DISCUSSION}

While skin color and its function in regulating UVR and discussed at a length in Anthropology. However, the characteristics of skin for example, structural integrity, thickness, developmental trajectories, and permeability against pathogens and allergens, have been studied primarily within biomedical context, without much appreciation for their evolutionary bases and anthropological implications. ${ }^{17}$ There is substantial evidence for selection effect to decrease pigmentation levels in both European and East Asian populations, and has not effected in a homogeneous pigmentation phenotype in either region. Some indigenous populations of northern Europe and Asia living at higher latitudes (and so experiencing lower UVR levels) have darker pigmentation than populations living further to the south. ${ }^{7}$ Earlier studies demonstrated a wide variation 
in skin colour in terms of Melanin Index in South Asia. Comparison with published datasets ${ }^{11}$ for the regions of the world revealed that the observed range in South Asians was three times greater than that in East Asians. Furthermore, the population from South India were found have remarkable variation in skin colour and the population from Northern and Western India demonstrated ${ }^{11-13}$ rather light skin coloured so far the Melanin Index concern. However, no comparative study was found among the population from Eastern and North East India. Nevertheless, between ethnicities, the most significant skin color differences occur along the yellowness $\left(\mathrm{b}^{*}\right)$ dimension in Mongoloid ethnic groups (Chakmas and Tripusis) in the present study ${ }^{18}$ is in consistency with earlier study.

\section{ACKNOWLEDGEMENTS}

Financial support has been provided University of Calcutta [BI (65) 8 \& 9] and UGC-UPE 2 Major Research Project grant. Authors are grateful to the participants and Dr. Rajesh Chatterjee, University of Tripura for his untiring help.

\section{REFERENCES}

1. Olson MV and Varki A. Sequencing the chimpanzee genome: insights into human evolution and disease. Nature Reviews Genetics 2003; 4: 20-28.

2. Varki A. Tasha $\mathrm{K}$ and Altheide TK. Comparing the human and chimpanzee genomes: Searching for needles in a haystack. Genetics Research 2000; 15: 1746-1758.

3. Serre $D$ and Pääbo $S$. Evidence for gradients of human genetic diversity within and among continents. Genetics Research 2004; 14: 1679-1685.

4. Laland KN, Odling-Smee $\mathrm{J}$ and Myles S. How culture shaped the human genome: Bringing genetics and the human sciences together. Nature Review Genetics 2010; 11: 137-148.

5. Walter $\mathrm{H}$. Remarks on the environmental adaptation of man. Human genetik 1971: 13: 85-97.
6. Roberts DF and Kahlon DPS. Environmental correlations of skin colour. Annals of Human Biology 1976; 3: 11-22.

7. Jablonski NG and Chaplin G. The evolution of human skin coloration. Journal of Human Evolution 2000; 39: 57-106.

8. Cann RL. Genetic clues to dispersal in human populations: retracing the past from the present. Science 2001; 291:1742-1748.

9. Bamshad MT, Kivisild WS, Watkins ME, Dixon CE, Ricker BB, Naidu JM, et al. Genetic evidence on the origins of Indian caste populations. Genome Research 2001; 11:994-1004.

10. Basu A, Sarkar-Roy N and Majumder PP. Genomic reconstruction of the history of extant populations of India reveals five distinct ancestral components and a complex structure. Proceedings of National Academy of Science 2016; 113: 201513197.

11. Basu Mallick C, lliescu F M, Möls M, Hill S, Tamang R, Chaubey G, et al. The light skin allele of SLC24A5 in South Asians and Europeans shares identity by descent. PLoS Genetics 2013; 9: e1003912.

12. Jonnalagadda $M$, Ozarkar $S$, Ashma $R$ and Kulkarni $S$. Skin pigmentation variation among populations of West Maharashtra, India. American Journal of Human Biology 2016; 1: 36-43.

13. Mishra A, Nizammuddin S, Mallick CB, Singh S, Prakash S, iddiqui NA, et al. Genotype-Phenotype Study of the Middle Gangetic Plain in India Shows Association of rs2470102 with Skin Pigmentation. Journal of Investigative Dermatology 2017; 3: 670-677.

14. CIE. Skin color analysis using a spectrophotometer in Asians. 1986 Austria: Central Bureau of the Commission Internationale de L'Eclairage.

15. Weatherall IL and Coombs BD. Skin color measurements in terms of CIELAB color space values. Journal of Investigative Dermatology 1992; 99: 468-473.

16. Seitz JC and Whitmore CG. Measurement of erythema and tanning responses in human skin using a tri-stimulus colorimeter Dermatologica 1988; 177:70-75.

17. Quillen EE, Norton HL, Parra EJ, Lona-Duraz F, Ang KC, Illiescu FM, et al. Shades of complexity: New perspectives on the evolution and genetic architecture of human skin. American Journal of Physical Anthropology 2019; 168: S67:4-26.

18. Xiao K, Yates JM, Zardawi F, Sueeprasan S, Liao N, Gill L, et al. Characterising the variations in ethnic skin colours: a new calibrated data base for human skin. Skin Research and Technology 2016; 0: 1-9.

\section{Authors Contribution:}

SM - Data Collection, data handling; DC - Concept and Design of the study, Review of Literature, Statistically analyzed; ARB - Concept and design, manuscript preparation, statistically analyzed and interpreted, critical revision of the manuscript.

Work attributed to:

Department of Anthropology, University of Calcutta

Orcid ID:

Prof. Arup Ratan Bandyopadhyay - (i) https://orcid.org/0000-0003-3119-0022

Source of Support: Financial support has been provided University of Calcutta [BI (65) 8 \& 9] and UGC-UPE 2 Major Research Project grant. Conflict of Interest: None. 